\title{
In silico study of anticarcinogenic potential of the selenoprotein BthD from Drosophila melanogaster. Identifying the anticancer peptide CRSUR from the conserved region
}

\author{
Toluwase Hezekiah Fatoki ${ }^{1,2, \bowtie}$, Omodele Ibraheem ${ }^{1}$, Amos Olalekan Abolaji ${ }^{3}$ \\ and David Morakinyo Sanni ${ }^{4}$
}

${ }^{I}$ Translational Bioinformatics Unit, Department of Biochemistry, Federal University Oye, PMB 373 Oye-Ekiti, Ekiti State, Nigeria

${ }^{2}$ Fhezt Bioinformatics Laboratory, Ifaki-Ekiti, Ekiti State, Nigeria

${ }^{3}$ Department of Biochemistry, Faculty of Basic Medical Sciences, College of Medicine, University of Ibadan, Oyo State, Nigeria

${ }^{4}$ Department of Biochemistry, Federal University of Technology Akure, PMB 704, Akure, Ondo State, Nigeria

\section{Article info}

\section{Article history:}

Received: $28^{\text {th }}$ February 2020

Accepted: $5^{\text {th }}$ May 2020

\section{Keywords:}

Anticancer peptide

Drosophila melanogaster

Mechanism design

NF-kappa-B signaling

Selenoprotein BthD

\begin{abstract}
Drosophila melanogaster is used as a model system in biomedical studies. Selenoprotein is the major biological form of selenium in eukaryotes. Selenoproteins are generally involved in catabolic pathways in bacteria and archaea, whereas it participates in anabolic and antioxidant processes in eukaryotic. In this study, anticancer potential of selenoprotein BthD of D. melanogaster was investigated using bioinformatics methods. Results showed that selenoprotein BthD of D. melanogaster may have dual properties as evident by its orthology with selenoprotein $\mathrm{H}$ (SelH) of Homo sapiens and conserved domain of fructokinase-like protein 2 of Vitis vinifera. These dual properties were also revealed in the phylogenetic analysis, while further structural modeling showed that selenoprotein BthD possibly exists as homotetramer in the native functional structure. The anticancer property of selenoprotein $\mathrm{BthD}$ was proposed to be by synergy of antioxidant or redox activities of thioredoxin and glutathione reductase (TGR) domain and the signaling function of fructokinase-like protein 2 domain both in Golgi apparatus and cytoplasm, through energy deprivation. The anticancer peptide CRSUR was identified from conserved region of selenoprotein BthD, of which its cyclic form showed potential anticancer properties predictively through E3 ubiquitin-protein ligase regulating NF-kappa-B signaling by unleashing cells for spontaneous formation of the ripoptosome.
\end{abstract}

(C) University of SS. Cyril and Methodius in Trnava

\section{Introduction}

The genomic sequence of D. Melanogaster is about $115229998 \mathrm{bp}$ and contains 13329 annotated genes (Adams et al. 2000). Drosophila possess genes systems which regulate nutrient uptake, storage and metabolism that are critical to survival and have been found conserved almost in all eukaryotes including humans, brown alga, zebrafish, mouse, Escherichia coli, and Caenorhabditis elegans (Allocca et al. 2018; Hatfield et al. 2014). D. melanogaster has been used as a model system for toxicological studies and diseases mechanism such as neurological 
disorders, developmental disorders, metabolic and storage disorders, cancer and cardiovascular disease (Bier 2005; Abolaji et al. 2014; Saraiva et al. 2018).

The major biological form of selenium in eukaryotes is selenocysteine ( $\mathrm{Sec}$ ) and its mostly found in the active site of selenoproteins. Selenocysteine is called the $21^{\text {st }}$ amino acid which has chemical structure differs from cysteine only by the presence of selenium in place of the sulfur atom. Sec is co-translationally inserted into a polypeptide chain in response to in-frame UGA codons directed by the $\mathrm{Sec}$ insertion sequence element, a stem-loop structure in the untranslated regions (3-UTRs) of selenoprotein mRNAs (Hatfield et al. 2014). The human genome contains 25 selenoprotein genes (Kryukov et al. 2003) and they are involved in a variety of functions, most notably redox homeostasis. Larger selenoproteomes can be found in aquatic organisms such as zebrafish, and brown alga (Lobanov et al. 2007; Hatfield et al. 2014). Selenoproteins are generally involved in catabolic pathways in bacteria and archaea, whereas eukaryotic selenoproteins participate rather in anabolic and antioxidant processes (Herbette et al. 2007). Selenoproteins participate in thyroid hormone metabolism, muscle formation, selenocysteine synthesis and in sperm maturation (Rederstorff et al. 2006). According to Gladyshev et al. (2016), selenoproteins without known functions include SELENOF (selenoprotein F, 15-kDa selenoprotein, SEP15), SELENOH (selenoprotein H, SELH, C11orf31), SELENOK (selenoprotein K, SELK), SELENOM (selenoprotein M, SELM), SELENOO (selenoprotein O, SELO), SELENOT (selenoprotein T, SELT), and others. Human selenoprotein enzymes with known functions such as thioredoxin reductases (TR1), glutathione peroxidases (Sep15 and GPx2) are important cellular redox-regulators needed by both normal and cancer cells, which result in anti- and protumorigenic effects at a tissue-specific cellular level (Hatfield et al. 2014). In liver TRI exhibits anticancer protein and in lung TR1 is a pro-cancer protein and a prime candidate for cancer therapy (Yoo et al. 2006; Carlson et al. 2012). The GPx1 polymorphisms are associated with cancer risk (Zhuo and Diamond 2009). It remains to be elucidated whether these anti- and pro-tumorigenic effects are tumor stage or grade-dependent.

The known selenoproteins in D. melanogaster are dSPS2, dSelK (former called dSelG) and dSelH (also known as dSelM or BthD) (HirosawaTakamori et al. 2000; Castellano et al. 2001). $\mathrm{dSelK}$ has one cysteine homolog and $\mathrm{dSelH}$ has two (Castellano et al. 2001; Martin-Romero et al. 2001). dSelH appears to belong to a class of selenoproteins widely distributed across the phylogenetic spectrum, as $\mathrm{dSelH}$ was found in zebrafish, human and mouse expressed sequence tag (EST) databases (Dickiy et al. 2007; Novoselov et al. 2007). The ability of dselH to reverse the toxic effects of glutathione depletion in Schneider cells was suggested to reflect a glutathione sparing effect via increased activity of an alternative anti-oxidant pathway, which restores the perturbed anti-oxidant-pro-oxidant balance (Morozova et al. 2003). Disruption of selenophosphate synthetase expression has recently been shown to modulate the Ras/MAPK signalling cascade in flies (Morey et al. 2001).

Ser/Thr kinase domain is one of the core kinase cascades in D. melanogaster and mammals (Yin and Zhang 2011). In D. melanogaster, Ser/Thr kinase domain is found in the core kinases of Hippo signaling pathway, as well as in the fourjointed and discs overgrown of upstream regulatory components (Yin and Zhang 2011). Defects in the core kinases and some of the upstream components of the pathway lead to robust organ overgrowth and link to numerous cancers (Pan 2010; Zhang et al. 2009). The Hippo signaling pathway limits organ size by regulating cell proliferation and apoptosis. In this study, anticancer potential of selenoprotein $\mathrm{BthD}$ of $D$. melanogaster was investigated based on the available genomic data and publications. The gene product with anticancer properties in an insect could be useful in the development of biologic agent for human cancer therapy.

\section{Experimental}

\section{Drosophila melanogaster selenoproteins}

The selenoprotein was searched from D. melanogaster database on Ensembl genome browser v97 (http://www.ensembl.org). The genes 
obtained from ensembl were looked up in the D. melanogaster database (www.flybase.org). The protein sequences and information were obtained from UniProt database (www.uniprot.org). The protein sequence of BthD of D. melanogaster was used to query Homo sapiens database on the Blastp server of NCBI (Camacho et al. 2009).

\section{Conserved protein domain}

Human selenoproteins TRI, Sep15 and GPx2 has been reported to possessed anti- and protumorigenic effect (Hatfield et al. 2014). The sequence of these proteins was obtained from UniProt. The conserved protein domain of all the selenoproteins of D. Melanogaster and four selenoproteins of Homo sapiens were investigated using the protein sequences on the CDD server (https://www.ncbi.nlm.nih.gov/Structure/cdd/wrpsb .cgi) of NCBI (Marchler-Bauer et al. 2017). The protein sequence of $\mathrm{BthD}$ of $D$. melanogaster was used to query database of eight plants in the taxonomy of Mesangiospermae identified from CDD results, on the Blastp server of NCBI (Camacho et al. 2009).

\section{Phylogenetics analysis}

The protein sequence of selenoproteins of $D$. melanogaster and $H$. sapiens obtained from the previous steps, were used for phylogenetics study. Multiple sequence alignment on ClustalO server (www.ebi.ac.uk/tools/msa/clustalo) was carried out and phylogenetic tree was constructed. The tree data was visualized at www.phylo.io.

\section{Structural modeling of selenoprotein BthD}

The three-dimensional structure of selenoprotein BthD of D. melanogaster was modelled on Swissmodel server (www.swissmodel.ch) using protein sequence (Camacho et al. 2009; Remmert et al. 2012; Waterhouse et al. 2018).

\section{Integration of anticarcinogenic mechanism} of selenoprotein BthD

The D. melanogaster pathways associated with growth of tumor were obtained from www.kegg.jp and available information in the scientific literatures was mined. These data were integrated with the key results of this study and anticarcinogenic mechanism of selenoprotein BthD of D. melanogaster was proposed.

\section{In silico prediction anticancer peptides}

of selenoprotein BthD and their physicochemical properties

The prediction of anticancer peptides in selenoprotein BthD was performed on AntiCP server Protein Scan (https://webs.iiitd.edu.in/raghava/anticp/submit_pro t.php), and generated the fragments amino acids residues of length of 5 with minimum Support vector machine (SVM) score of 1.15 , and predict their anticancer property along with all

Table 1. Details of selenoprotein genes, and proteins of D. melanogaster integrated from Ensembl, Flybase and Uniprot.

\begin{tabular}{|c|c|c|c|c|c|c|c|c|}
\hline $\begin{array}{l}\text { Gene } \\
\text { name }\end{array}$ & $\begin{array}{l}\text { Chromosome } \\
\text { location }\end{array}$ & $\begin{array}{l}\text { No. of } \\
\text { transcript }\end{array}$ & $\begin{array}{l}\text { Human orthologs } \\
\text { [specieslgene } \\
\text { symbol] }\end{array}$ & $\begin{array}{l}\text { Best } \\
\text { transcript } \\
\text { name and ID }\end{array}$ & $\begin{array}{l}\text { Length } \\
\text { [nt] }\end{array}$ & $\begin{array}{l}\text { Uniprot } \\
\text { ID }\end{array}$ & $\begin{array}{l}\text { Length } \\
\text { [amino } \\
\text { acids] }\end{array}$ & $\begin{array}{l}\text { Subcellular } \\
\text { location }\end{array}$ \\
\hline BthD & $\begin{array}{l}\text { Chr. X: } \\
13,612,131- \\
13,613,228\end{array}$ & 1 & HsaplSELENOH & $\begin{array}{l}\text { BthD-RA, } \\
\text { FBtr0073806 }\end{array}$ & 977 & Q9VYB0 & 249 & Cytoplasm \\
\hline SelG & $\begin{array}{l}\text { Chr. X: } \\
11,887,284- \\
11,888,24\end{array}$ & 1 & Hsap\SELENOK & $\begin{array}{l}\text { SelG-RA, } \\
\text { FBtr0073570 }\end{array}$ & 827 & Q7Z2C4 & 110 & Golgi apparatus \\
\hline SelR & $\begin{array}{l}\text { Chr. 3R: } \\
\text { 10,863,355- } \\
10,868,642\end{array}$ & 8 & Hsap\MSRB3 & $\begin{array}{l}\text { SelR-RE, } \\
\text { FBtr0082295 }\end{array}$ & 1156 & $\begin{array}{l}\text { Q8INK9, } \\
\text { D3DMP0 }\end{array}$ & 208,192 & $\begin{array}{l}\text { Nucleus, } \\
\text { Cytoplasm, cyto- } \\
\text { skeleton }\end{array}$ \\
\hline SelT & $\begin{array}{l}\text { Chr. 2L: } \\
5,010,922- \\
5,12,127\end{array}$ & 3 & Hsap\SELENOT & $\begin{array}{l}\text { SelT-RA, } \\
\text { FBtr0079000 }\end{array}$ & 960 & $\begin{array}{l}\text { M9PCL4, } \\
\text { Q9VMV6 }\end{array}$ & 198 & $\begin{array}{l}\text { Integral component } \\
\text { of membrane, } \\
\text { endomembrane } \\
\text { system }\end{array}$ \\
\hline
\end{tabular}



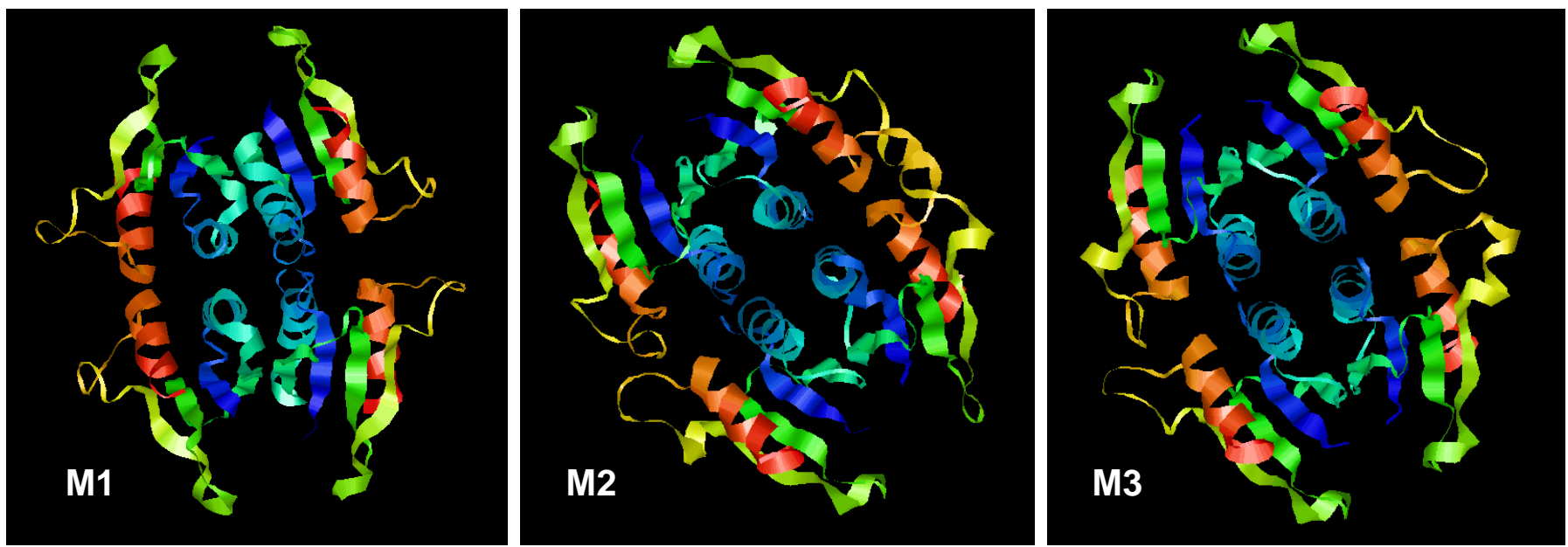

Fig. 1. Model structures of selenoprotein BthD of D. melanogaster in homotetramer oligomeric state.

the important physico-chemical properties
like hydrophobicity, charge, pI etc. The cell-penetrating efficacy of peptides were predicted on CPPred-RF server (http://server.malab.cn/CPPred-RF/index.jsp) (Wei et al. 2017).

In silico target prediction and pharmacokinetics of anticancer peptides of selenoprotein BthD

The structure and SMILES (Simplified Molecular Input Line Entry Specification) format of selenocysteine-containing peptide (in cysteine form) was obtained from PepSMI server (https://www.novoprolabs.com/tools/convert-

peptide-to-smiles-string) and cysteine residue was edited to selenocysteine on https://pubchem.ncbi.nlm.nih.gov/edit3/index.html. In silico prediction of target was done on SwissTargetPrediction server, where Homo sapiens was selected as target organism (Diana et al. 2019). The active peptides (peptides with predicted target) were then subjected to in silico Absorption, Distribution, Metabolism, and Excretion (ADME) screening on SwissADME server at default parameters. (Diana et al. 2017).

\section{Results}

The search on the Ensembl genome browser showed four genes of selenoproteins present in the D. melanogaster genome. The gene products, orthology in $H$. sapiens and subcellular location were obtained from Flybase and UniProt databases, as shown in Table 1. Each of the four selenoproteins in D. melanogaster has one ortholog in human. The homology alignment of $\mathrm{BthD}$ protein sequence (Uniprot ID: Q9VYB0) by Blastp confirmed $41.38 \%$ similarity with human SelH (C11orf31) selenoprotein (Uniprot ID: Q8IZQ5) which is located in the Golgi apparatus and cytoplasm.

The conserved domain of $D$. melanogaster and $H$. sapiens are summarized in Table 2. The conserved domain of selenoprotein BthD was found to be exceptional with no similarity in $H$. sapiens unlike the other three. The conserved protein domain of BthD was classified as kinase which belong to PLN02967 superfamily of fructokinase-like protein 2 (EC 2.7.1.4), and belong to protein clusters conserved in taxonomy of Mesangiospermae in eukaryotic plant. This study is the first to discover and report the plant-like properties of $\mathrm{BthD}$. Further homology alignment of the BthD protein sequence against eight plants in the PLN02967 superfamily of taxonomy Mesangiospermae, only showed $33.33 \%$ similarity to an uncharacterized selenoprotein $\mathrm{H}$ (UniProt ID: D7SU28) of Vitis vinifera.

Moreover, human SelH contained domain architecture which is similar to that SelT of D. melanogaster and has been noted as thioredoxin and glutathione reductase (TGR Domain). This domain is homodimeric, FADcontaining member of the pyridine nucleotide disulfide oxidoreductase family. Table 3 shows the summary of homology analysis of BthD protein 
Nova Biotechnol Chim (2020) 19(1): 37-51

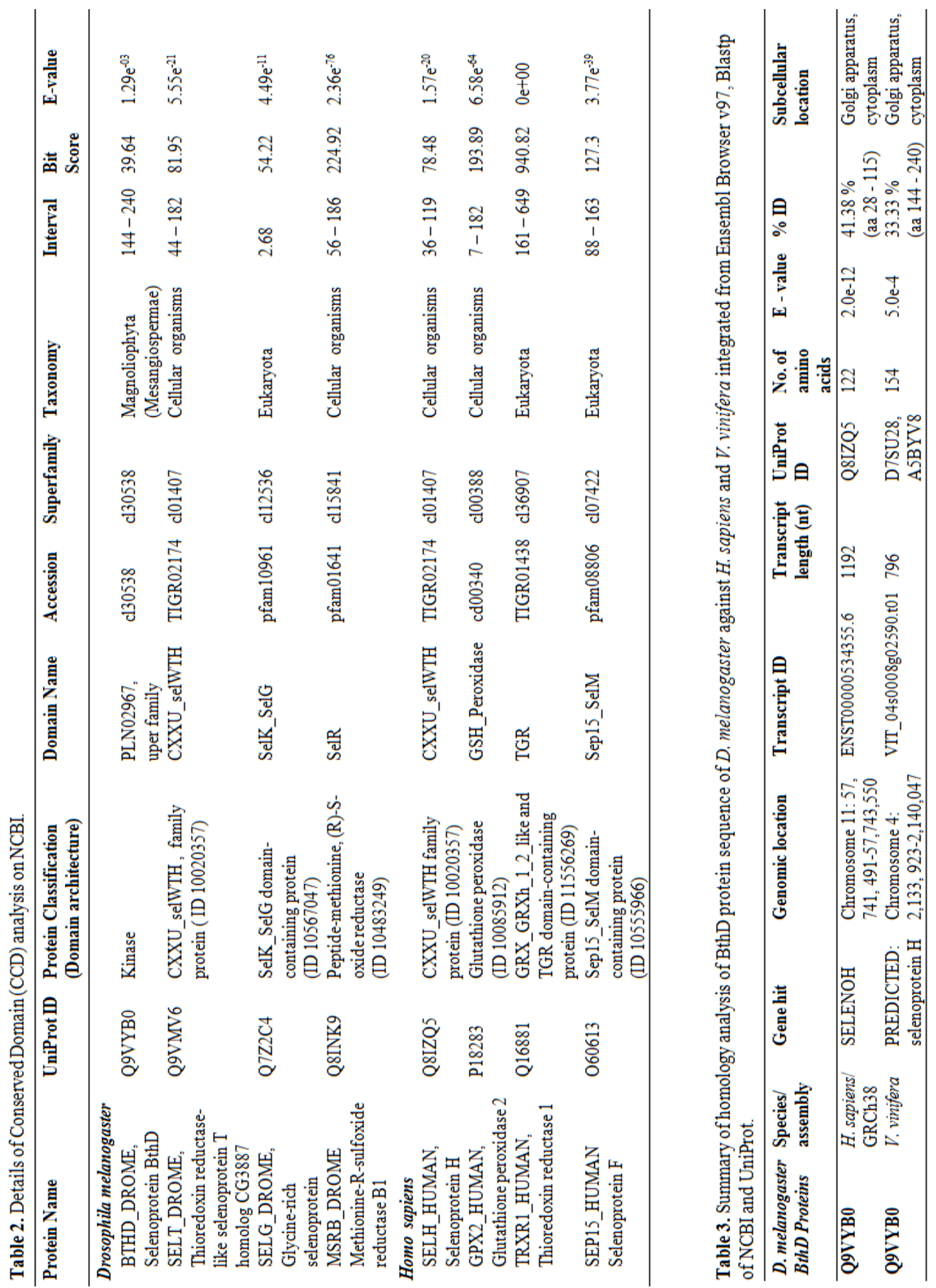




SP|Q9VMV6 |SELT_DROME SP|Q8INK9|MSRB_DROME 5P|P18283|GPX2 HUMAN sp|060613|SEP15_HUMAN SP $\mid$ Q722C4|SELG_DROME Sp |Q16881|TRXR1_HUMAN tr|D7SU28|D7SU28.VITVI sp|Q9VYBe|BTHD_DRिOME sp |Q8IZQS |SELH_HUMAN

SP|Q9VMVG ISELT DROME SP|Q8INK9|MSRB_DROME SP|P18283|GPX2_HUMAN SP|O60613 |SEP15 HUMAN SP|0772C4|SELG DROME SP|Q16881| TRXR 1 HUMAN tr|D7SU28|D7SU28_VITVI Sp|Q9VYB]|BTHD_DROME SP|Q8IZQ5|SELH_HUMAN

SP|Q9VMV6 |SELT_DROME SP|Q8INK9|MSRB_DROME Sp|P18283|GPX2 HUMAN sp|060613|SEP15 HUMA: Sp|Q7Z2C4|SELG_DROME SP|Q16881|TRXR_1_HUMAN tr|D7SU28|D7SU28 VITVI Sp|Q9VYBB|BTHD DROME SP|Q8IZQS | SELH_HUMAN

SP|Q9VMV6|SELT_DROME SP|Q8INK9|MSRB_DROME SP|P18283|GPX2 HUMAN Sp|Обеб13|SEP15_HUMAN SP|Q7Z2C4|SELG_DROME SP|Q16881|TRXR1 HUMAN tr|D7SU28|D7SU28 VITVI SP|Q9VYBO|BTHD_DROME SPIQSIZQS |SELH_HUMAN

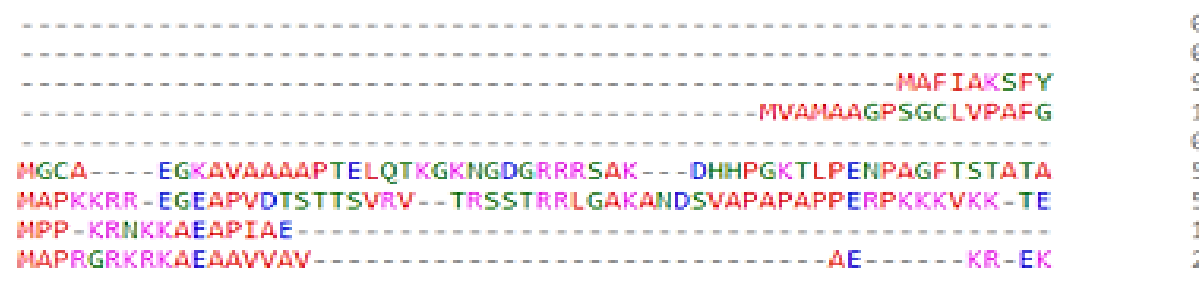

.... MERL TGRNNALLVLCLCAG-YALVFAEGEKEIPVTKFGONIAPTMTF D. DLSAISLDGEKVDFNTFRGRAVL IENVASLUGTTTRDFTQ-LNELQCR _... _. FPRRLV -LR . . . . . . . . . . . LLLATVLQAVSAFGAEFSS-EAC . . R . . . ELGFSSN . DSR - . . . . . . . . . ALLQAYIDGHS - -VVIFSR-STCTRCT_... EVKKLFK 87 DVK _... . . . . . . EPEKVADGSK - T - IVI - EHCKQCN _ . . - SFKTRAT 87

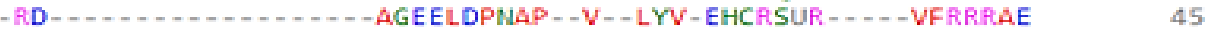

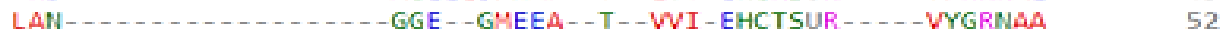

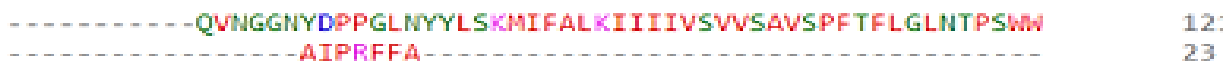
LVQKC - . EVNGQNEHPV FA FA E

AFVRS . . . .

AFVRS - $\ldots \ldots \ldots \ldots$. 17



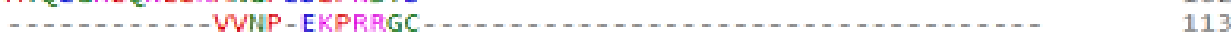
QLNAL-GAPRRGA $\ldots \ldots \ldots \ldots$

KV-NP-TKPRRGS ............ 78

SHMQANKIYACMMIFFLGNMLEAQLISSGAFEITLNDWPVWSK - LQTGRFPSPEVLF - -Q 178 -DSRQD- - SDNP - . - .

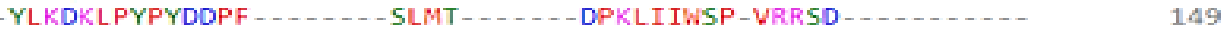

GLQIKYVRGSDPVL . . . . . . KLLDONGNIAEELSILKWNT-DSVEEFLSEKL _. . . . 162

.

191

144

116

112

Fig. 2. Multiple sequence alignment of all selenoproteins of D. melanogaster, four selenoproteins of $H$. sapiens and a selenoprotein of $V$. vinifera.

sequence of $D$. melanogaster against $H$. sapiens and $V$. vinifera integrated from Ensembl Browser v97, Blastp of NCBI and UniProt. The structural model parameter of BthD selenoprotein on Swissmodel server is shown in Table 4. The structure was modeled as homotetramer based on the percentage similarities to three different template proteins; 2ojl (crystal structure of Q7WAF1_BORPA from Bordetella parapertussis), 2obk (crystal structure of the putative Se binding protein from Pseudomonas fluorescens), and 2oka (crystal structure of Q9HYQ7_PSEAE from Pseudomonas aeruginosa) as shown in Fig. 1. This structure can be further elucidated through X-ray crystallography. The result in Fig. 2 showed the position of conserved amino acid residues among selenoproteins (D7SU28_ VITVI, BTHD_DROME and SELH_HUMAN). The phylogenetic tree confirmed the evolutionary relatedness of BthD gene of D. melanogaster, SelH gene of $H$. sapiens and D7SU28 gene of $V$. vinifera (Fig. 3).
The integral mechanism of anticarcinogenic property of selenoprotein BthD is shown in Fig. 4. This mechanism was based on the glycolytic energy stress induced by hepatocytes depletion of ATP by fructose and its impact of AMPK1, Hippo signaling and Notch1 signaling pathways; the role of trehalose to regulate glucose metabolism in stress condition; the ability of isomaltose to regulate adenylate biosynthesis; and the capacity $V$. vinefera to provide fructose and phytochemicals such as resveratrol. Based on the mechanism proposed in this study, we have hypothesized that amino acid sequences EHCRSUR and GAPRRGA from selenoprotein BthD as well as EHCKQCN and EKPRRGC from $V$. vinefera (grape) could be the key bioactive peptides that can alone and synergistically modulate this mechanism and impact the required antioxidant effect. This was validated by the results of anticancer peptides from selenoprotein BthD shown in Table 5, where selenocysteine-containing peptide CRSUR has SVM score of 1.37 and high 


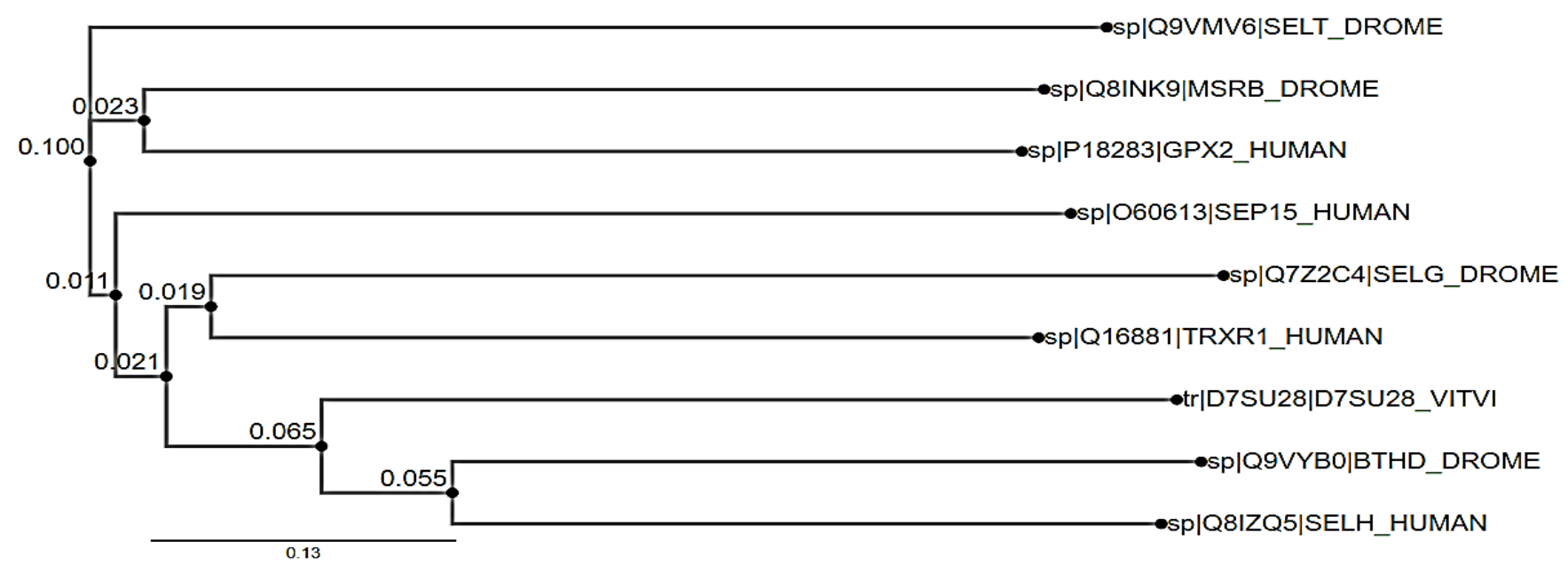

Fig. 3. The phylogenetic tree of all selenoproteins of $D$. melanogaster, four selenoproteins of $H$. sapiens and a selenoprotein of $V$. vinifera.

Cell-penetrating uptake efficiency, while RRGAF has SVM score of 1.28 and high cell-penetrating uptake efficiency. The structure of the CRSUR peptide in linear and cyclic (head-to-tail bond) is shown in Table 6. The predicted targets of CRSUR peptide from selenoprotein BthD show that this selenocysteine-containing peptide have anticancer and antiviral or antineuronal properties based on the biological processes by the target genes obtained such as Furin, Integrin beta3, andBaculoviral IAP repeat-containing protein (Table 7). The result of pharmacokinetics of CRSUR (Table 8) indicate that gastrointestinal route will not be good for the administration of bioactive peptide.

\section{Discussion}

Selenoprotein BthD (BthD) has been reported to possesses antioxidant potential (Castellano et al. 2001). Selenoprotein T (SelT) is a thioredoxindisulfide reductase (EC 1.8.1.9) that belongs to the SelWTH family and SELT subfamily. Selenoprotein R (SelR or MsrB) is a peptidemethionine (R)-S-oxide reductase (EC 1.8.4.12) which belongs to the MsrB Met sulfoxide reductase family (Kryukov et al. 2003). The thioredoxin and glutathione reductase (TGR Domain) is homodimeric, FAD-containing member of the pyridine nucleotide disulfide oxidoreductase family which contains a C-terminal motif CysSeCys-Gly, where SeCys is selenocysteine encoded by TGA which is a stop codon in some sequence).
(Sun et al. 2001 TIGR02174 domain is a member of the superfamily cl01407 together with related to pfam10262, a domain found in both bacteria and animals selenoproteins SelT, SelW, and SelH (Dickiy et al. 2007).

Taxonomy of Mesangiospermae belongs to eukaryotic plant and its PLN02967 superfamily was similar to an uncharacterized selenoprotein $\mathrm{H}$ (UniProt ID: D7SU28) of $V$. vinifera. Some studies have reported that grape ( $V$. vinifera) extracts showed cytotoxicity towards cultured cells as well as inhibited tumor growth in animal models (Shrotriya et al. 2012; Sun et al. 2012).

Different molecular mechanisms have been proposed for these protective effects of grape extracts, such as inhibition of enzymes playing an essential role in cell proliferation (e.g. human topoisomerase I) and inhibition of angiogenesis (Agarwal et al. 2004; Stagos et al. 2005). The result of a double-blinded randomized crossover human trial showed that dietary supplementation of grape seed extract at a dose of $600 \mathrm{mg} /$ day for 4 weeks can decrease of oxidative stress and enhance glutathione (GSH)/oxidized glutathione (GSSG) and total antioxidant status (Kar et al. 2009). The anticancer effects of whole black grape (seeds included) extract have been reported in the cancerous colon tissues of humans by inhibition in DNA turnover enzymes (Durak et al. 2005). An in silico study has reported the molecular targets for the key bioactive components present in grape such as resveratrol, piceatannol, and scirpusin A (Fatoki et al. 2018a). 


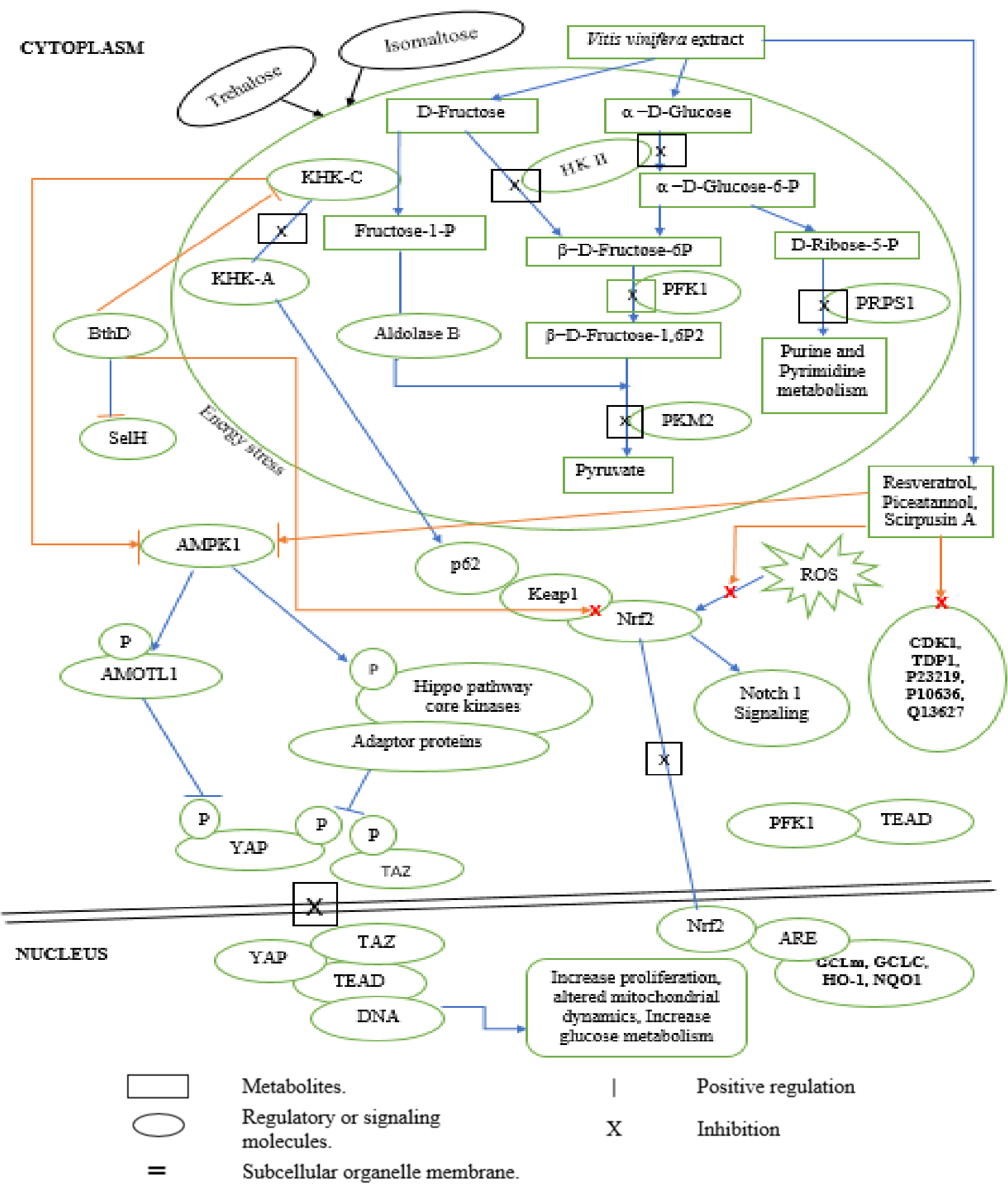

Fig. 4. Integrated anticarcinogenic mechanism of Selenoprotein BthD.

Comprehensive review of anticancer properties of grape can be found in another publication (Zhou and Raffoul 2012).

In D. melanogaster, three forms of hexokinases (Hex A, B and C) were found by agar gel electrophoresis (Madhavan et al. 1972). Hex A investigated. The chromosomal location of BthD is and Hex $B$ have been mapped on the same structural gene on the $\mathrm{X}$ chromosome (Voelker et al. 1978) while Hex C on the second chromosome (Jelnes 1971). However, whether these three hexokinases were either aldose or ketose was not 
Table 6. Structure of the peptide CRSUR Linear and Head-to-tail bond.

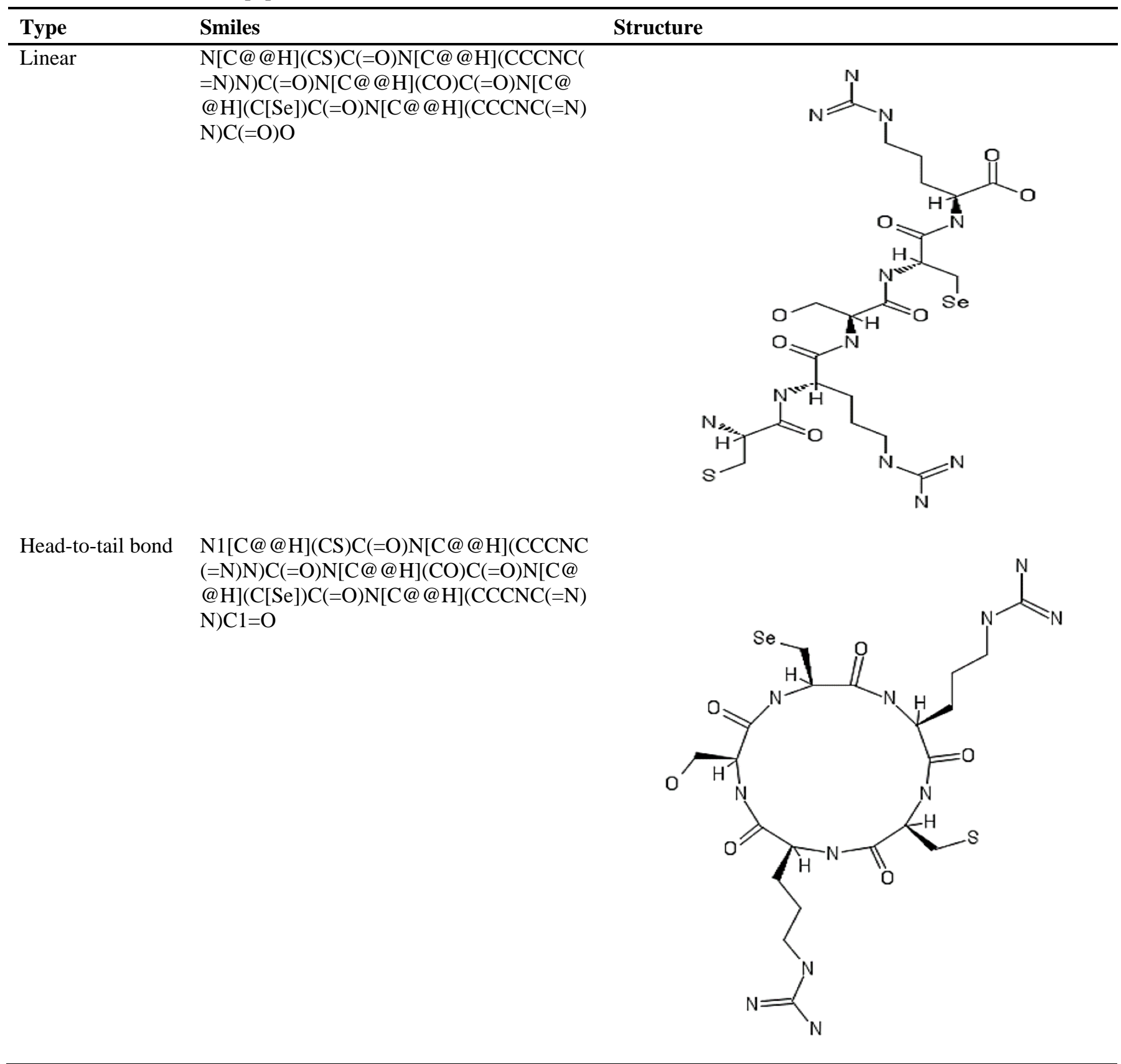

Chromosome X: 13,612,131-13,613,228 (Table 1). which inhibited fructose-mediated cytoprotection Fructokinase (also known as ketohexokinase; (Speicher et al. 2010).

KHK), which catalyzes the phosphorylation Study has shown that trehalose do not stimulate of fructose to fructose 1-phosphate, was identified by MALDI-TOF MS and found expressed at extremely low rates in the renal tumor tissues (Hwa et al. 2006). A study has shown that fructoseinduced ATP depletion in human, rat and mouse hepatocytes cause full protected against tumor necrosis factor-alpha (TNF- $\alpha)$-induced cytotoxicity, whereas hepatic tumor cell lines showed increased hexokinase II (HKII) expression rapid increases in blood glucose and excessive secretion of insulin and gastric inhibitory polypeptide (GIP) promoting fat accumulation (Yoshizane et al. 2017). It has been demonstrated that trehalose-6-phosphate (T6P) inhibits yeast hexokinase 2 (HKII) activity, thus it is likely that this metabolite regulates glycolysis by modulating the flow of phosphorylated sugars towards this pathway (Blázquez et al. 1993; 
Table 7. Predicted targets of CRSUR Peptide from selenoprotein BthD.

\begin{tabular}{lllcc}
\hline & & & \multicolumn{2}{c}{$\begin{array}{c}\text { CRSUR Peptide } \\
\text { [\% probability] }\end{array}$} \\
\cline { 3 - 5 } & Targets & UniProt ID & Linear & Head-to-tail bond \\
\hline 1 & Furin & P09958 & 60 & 45 \\
2 & Proprotein convertase subtilisin/kexin type 4, 5, 6 & Q6UW60, Q92824, P29122 & 60 & 45 \\
3 & Complement factor B Ba fragment & P00751 & 55 & - \\
4 & Complement C2 & P06681 & 55 & - \\
5 & Neurotensin receptor type 1, 2 & P30989, O95665 & 50 & - \\
6 & WD repeat-containing protein 5, 5B & P61964, Q86VZ2 & 40 & 45 \\
7 & Coagulation factor VII, IXa heavy chain & P08709, P00740 & 40 & 40 \\
8 & Factor X light chain & P00742 & 40 & 40 \\
9 & Complex (Integrin beta-3) & P08514/P05106 & 35 & 50 \\
10 & E3 ubiquitin-protein ligase XIAP & P98170 & - & 45 \\
11 & Baculoviral IAP repeat-containing protein 2, 3,8 & Q13490, Q13489, Q96P09 & - & 45 \\
\hline
\end{tabular}

Probability on target was computed based on a cross-validation. They may therefore not represent the actual probability of success for any new molecule.

Thevelein et al. 1995). T6P plays a critical role as a sensing molecule that promotes sugar fermentation and glucose repression in yeast (Vicente et al. 2018). Thus, trehalose diet by cancer patient may be necessary to trigger energy stress which will in turn open up cancer cell to the pathway of selenoprotein BthD. Isomaltose will be needed to mitigate against the diabetes signaling molecules that are associated with cancer cell proliferation. Isomaltose inhibit adenylosuccinate lyase which is a more proximal enzyme in the adenylosuccinate biosynthesis pathway, lowers S-AMP levels and impairs glucosestimulated insulin secretion (Fatoki et al. 2018b), and may help to reduce the risks associated with obesity and type 2 diabetes (van Can et al. 2012).

Previous studies haves established that phosphoglycerate kinase 1 (PGK1) and pyruvate kinase M2 (PKM2) the only two ATP-generating glycolytic enzymes, which function as protein kinases and play active roles in tumor development (Li et al. 2016a). Another study has shown that hepatocellular carcinoma (HCC) cells reduce the fructose metabolism rate, and involved a switch in expression from fructokinase $\mathrm{C}$ (KHK-C) to fructokinase A (KHK-A) (Li et al. 2016b), and in the process KHK-A enhanced nucleic acid synthesis for tumorigenesis ( $\mathrm{Li}$ et al. 2016c), and also enhanced p62's aggregation with Kelchlike ECH-associated protein 1 (Keap1) and nuclear factor erythroid 2-related factor 2 (Nrf2) activation (Xu et al. 2019). Nrf2 is located in the cytoplasm and guided by Keap1, but under oxidative stress
Nrf2 moves to the nucleus, where it binds the antioxidant response element (ARE) and drives the expression of several downstream genes such as $\gamma$-glutamyl cysteine synthetase modifier subunit (GCLm), glutamate cysteine ligase catalytic subunit (GCLC), heme oxygenase-1 (HO-1) and $\mathrm{NAD}(\mathrm{P}) \mathrm{H}$ quinine oxidoreductase-1 (NQO1) (Shibata et al. 2008; Singh et al. 2008; Ding et al. 2010; Kansanen et al. 2013). A novel peptide activator of a key antioxidant gene transcription pathwayin the hippocampus, which disrupted the Nrf2-Keap1 interaction in global cerebral ischemia model has been reported (Tu et al. 2015). The Hippo signaling pathway plays a crucial role in cell proliferation, apoptosis, differentiation, and development. Transcriptional co-activators Yes-associated protein 1 (YAP) and WW domaincontaining transcription regulator protein 1 (TAZ), are major effectors of the Hippo signaling pathway (Bae et al. 2017). They function as transcription factors along with TEAD (TEA domain family member) in the nucleus, which increases expression of such target genes as Ctgf, Cyr61, AXL, and Survivin (Bae et al. 2017). Study has shown that phosphofructokinase 1 (PFK1) mediates glucose-induced YAP- and TAZ-TEAD interactions (Enzo et al. 2015). Energy stress, as induced by culturing cells in glucose-free conditions, results in inhibition of YAP activity in mouse hepatocytes in vivo as demonstrated by starvation/re-feeding experiments (Wang et al. 2015). In recent time, small peptides having anticancer properties have emerged as a potential 
Table 8. Pharmacokinetics of CRSUR Peptide from Selenoprotein BthD.

\begin{tabular}{llcc}
\hline & \multicolumn{1}{c}{ Parameters } & \multicolumn{2}{c}{ CRSUR Peptide } \\
\cline { 3 - 4 } & & Linear & Head-to-tail bond \\
\hline 1 & Molecular Weight [g.mol ${ }^{-1}$ ] & 669.64 & 651.62 \\
2 & Heavy Atoms (HA) & 41 & 40 \\
3 & Molar Refractivity & 151.53 & 167.52 \\
4 & Total Polar Surface Area (Å2) & 362.55 & 328.33 \\
5 & Lipophilicity Consensus LogP & -3.96 & -3.73 \\
6 & Water Solubility ESOL Class & Highly soluble & Very soluble \\
7 & Gastrointestinal Absorption & Low & Low \\
8 & Blood Brain Barrier (BBB) Permeant & No & No \\
9 & P-glycoprotein Substrate & No & No \\
10 & Cytochrome P450s Inhibitor & No & No \\
11 & Skin permeation log Kp [cm.s ${ }^{-1}$ ] & -15.29 & -12.84 \\
12 & Lipinski Violation & 3 & 3 \\
13 & Bioavailability Score & 0.17 & 0.17 \\
14 & Synthetic Accessibility & 5.62 & 6.40 \\
\hline
\end{tabular}

alternative approach for cancer therapy (Thundimadathil et al. 2012). Anticancer peptides (ACPs) are small (5 - 30 amino acids) peptides, often derived from antimicrobial peptides (AMPs) and are cationic in nature (Tyagi et al. 2013), while cell-penetrating peptides (CPPs) are small peptides that have unique inherent ability to directly enter cells without significantly damaging the cell membrane (Wei et al. 2017). In this study, the selenocysteine-containing peptide CRSUR was identified and further investigated among all peptides obtained. The results show that cyclic peptide CRSUR will be a good anticancer agent through E3 ubiquitin-protein ligase regulating NFkappa-B signalling by unleashes cell for spontaneous formation of the ripoptosome, a large multi-protein complex that has the capability to kill cancer cells in a caspase-dependent and caspaseindependent manner (Bertrand et al. 2011).

\section{Conclusions}

Low calories diets can cause significant reduction in tumor incidence and tumor growth, and the mechanistic links between diet and cancer which has remain poorly understood (Warr et al. 2018), has been unravelled for application in human health through this in-silico study. We have shown that selenoprotein BthD can be good antioxidant supplement alone or together with the whole fruit juice of $V$. vinifera, not only against cancer but also virus infection and for overall human health (Moghadaszadeh and Beggs 2006).
Thus, it is possible to build on the understanding of the antioxidant/anticancer potential of BthD by investigating new synthetic peptides from the conserved regions. Further study will be to evaluate anticancer potential of optimized peptides of selenoprotein $\mathrm{BthD}$ consisting of $5-10$ amino acid residues; and also investigate the Seccontaining disaccharides as novel anticancer compounds.

\section{Conflict of Interest}

The authors declare that they have no conflict of interest.

\section{References}

Abolaji AO, Kamdem JP, Lugokenski TH, Nascimento TK, Waczuk EP, Farombi EO, Loreto EL, Rocha JB (2014) Involvement of oxidative stress in 4 vinylcyclohexeneinduced toxicity in Drosophila melanogaster. Free Radic. Biol. Med. 71: 99-108.

Adams MD, Celniker SE, Holt RA, Evans CA, Gocayne JD, Amanatides PG, Scherer SE, Li PW, Hoskins RA, Galle RF (2000) The genome sequence of Drosophila melanogaster. Science 287: 2185-2195.

Agarwal C, Singh RP, Dhanalakshmi S, Agarwal R (2004) Anti-angiogenic Efficacy of grape seed extract in endothelial cells. Oncol. Rep. 11: 681-685.

Allocca M, Zola S, Bellosta P (2018) The fruit fly, Drosophila melanogaster: Modeling of human diseases (Part II). In Perveen FK (Ed.) Drosophila melanogaster Model for recent advances in genetics and therapeutics, Intechopen, pp. 131-156.

Bae JS, Kim SM, Lee H (2017) The Hippo signaling pathway provides novel anti-cancer drug targets. Oncotarget. 8: 16084-16098. 
Bertrand MJ, Lippens S, Staes A, Gilbert B, Roelandt R, De Medts J, Gevaert K, Declercq W, Vandenabeele P (2011) cIAP1/2 are direct E3 ligases conjugating diverse types of ubiquitin chains to receptor interacting proteins kinases 1 to 4 (RIP1-4). PLoS One. 6: e22356.

Bier E (2005) Drosophila, the golden bug, emerges as a tool for human genetics. Nat. Rev. Genet. 6: 9-23.

Blázquez MA, Lagunas R, Gancedo C, Gancedo JM (1993) Trehalose-6-phosphate, a new regulator of yeast glycolysis that inhibits hexokinases. FEBS Lett. 329: 5154.

Camacho C, Coulouris G, Avagyan V, Ma N, Papadopoulos J, Bealer K, Madden TL (2009) BLAST+: architecture and applications. BMC Bioinfomatics 10: 421-430.

Carlson BA, Yoo MH, Tobe R, Mueller C, Naranjo-Suarez S, Hoffmann VJ, Gladyshev VN, Hatfield DL (2012) Thioredoxin reductase 1 protects against chemically induced hepatocarcinogenesis via control of cellular redox homeostasis. Carcinogenesis 33: 1806-1813.

Castellano S, Morozova N, Morey M, Berry MJ, Serras F, Corominas M, Guigo R (2001) In silico identification of novel selenoproteins in the Drosophila melanogaster genome. EMBO Rep. 2: 697-702.

Diana A, Michielin O, Zoete V (2017). SwissADME: a free web tool to evaluate pharmacokinetics, druglikeness and medicinal chemistry friendliness of small molecules. Sci. Rep. 7: 42717.

Diana A, Michielin O, Zoete V (2019). SwissTargetPrediction: updated data and new features for efficient prediction of protein targets of small molecules. Nucleic Acids Res. 47: W357-W364.

Dickiy A, Novoselov SV, Fomenko DE, Sengupta A, Carlson BA, Cerny RL, Ginalski K, Grishin NV, Hatfield DL, Gladyshev VN (2007) SelT, SelW, SelH, and Rdx12: genomics and molecular insights into the functions of selenoproteins of a novel thioredoxin-like family. Biochemistry 46: 6871-6882.

Ding M, Zhao J, Bowman L, Lu Y, Shi X (2010) Inhibition of AP-1 and MAPK signaling and activation of Nrf2/ARE pathway by quercitrin. Int. J. Oncol. 36: 59-67.

Durak I, Cetin R, Devrim E, Erguder IB (2005) Effects of black grape extract on activities of DNA turn-over enzymes in cancerous and non-cancerous human colon tissues. Life Sci. 76: 2995-3000.

Enzo E, Santinon G, Pocaterra A, Aragona M, Bresolin S, Forcato M, Grifoni D, Pession A, Zanconato F, Guzzo G, Bicciato S, Dupont S (2015) Aerobic glycolysis tunes YAP/TAZ transcriptional activity. EMBO J. 34: 13491370.

Fatoki TH, Awofisayo OA, Ogunyewo OA, Ugboko HU, Sanni DM (2018a) Impacts of analogy and dimerization of bioactive compounds on molecular biological functions. J. Adv. Med. Pharm. Sci. 19: 45211.

Fatoki TH, Sanni DM, Momodu DU, Ugboko HU, Adeseko CJ, Faleye BC (2018b) Evaluation of empirical functions and fate of isomaltose. J. Appl. Life Sci. Int. 16: 9370.

Gladyshev VN, Arnér ES, Berry MJ, Brigelius-Flohé R, Bruford EA (2016) Selenoprotein gene nomenclature. J. Biol. Chem. 291: 24036-24040.
Hatfield DL, Tsuji PA, Carlson BA, Gladyshev VN (2014) Selenium and selenocysteine: roles in cancer, health and development. Trends Biochem. Sci. 39: 112-120.

Herbette S, Roeckel-Drevet P, Drevet JR (2007) Selenoindependent glutathione peroxidases. More than simple antioxidant scavengers. FEBS J. 274: 2163-2180.

Hirosawa-Takamori M, Jackle H, Vorbruggen G (2000) The class 2 selenophosphate synthetase gene of Drosophila contains a functional mammalian-type SECIS. EMBO Rep. 1: 441-446.

Hwa JS, Kim HJ, Goo BM, Park HJ, Kim CW, Chung KH, Park HC (2006) The expression of ketohexokinase is diminished in human clear cell type of renal cell carcinoma. Proteomics 6: 1077-1084.

Jelnes JE (1971) Identification of hexokinases and localisation of a fructokinase and a tetrazolium oxidase locus in Drosophila melanogaster. Hereditas 67: 291-293.

Kansanen E, Kuosmanen SM, Leinonen H, Levonen AL (2013) The Keap1-Nrf2 pathway: Mechanisms of activation and dysregulation in cancer. Redox Biol. 1: 45-49.

Kar P, Laight D, Rooprai HK, Shaw KM, Cummings M (2009) Effects of grape seed extract in Type 2 diabetic subjects at high cardiovascular risk: a double-blind randomized placebo-controlled trial examining metabolic markers, vascular tone, inflammation, oxidative stress and insulin sensitivity. Diabetic Med. 26: 526-531.

Kryukov GV, Castellano S, Novoselov SV, Lobanov AV, Zehtab, O, Guigó R, Gladyshev VN (2003) Characterization of mammalian selenoproteomes. Science 300: 1439-1443.

Li X, Jiang Y, Meisenhelder J, Yang W, Hawke DH, Zheng Y, Xia Y, Aldape K (2016a) Mitochondria-translocated PGK1 functions as a protein kinase to coordinate glycolysis and the TCA cycle in tumorigenesis. Mol. Cell. 61: 705-719.

Li X, Qian X, Peng LX, Jiang Y, Hawke DH, Zheng Y, Xia Y (2016b) A splicing switch from ketohexokinase-C to ketohexokinase-A drives hepatocellular carcinoma formation. Nat. Cell. Biol. 18: 561-571.

Li X, Qian X, Lu Z (2016c) Fructokinase A acts as a protein kinase to promote nucleotide synthesis. Cell Cycle 15: 2689-2690.

Lobanov AV, Fomenko DE, Zhang Y, Sengupta A, Hatfield DL, Gladyshev VN (2007) Evolutionary dynamics of eukaryotic selenoproteomes: large selenoproteomes may associate with aquatic life and small with terrestrial life. Genome Biol. 8: R198.

Madhavan K, Fox DJ, Ursprung H (1972) Developmental genetics of hexokinase isoenzymes in Drosophila melanogaster. J. Insect Physiol. 18: 1523-1530.

Marchler-Bauer A, Bo Y, Han L, He J, Lanczycki CJ, Lu S, Chitsaz F, Derbyshire MK, Geer RC (2017) CDD/SPARCLE: functional classification of proteins via subfamily domain architectures. Nucleic Acids Res. 45: 200-203.

Martin-Romero FJ, Kryukov GV, Lobanov AV, Carlson BA, Lee BJ, Gladyshev VN, Hatfield DL (2001) Selenium 
metabolism in Drosophila: selenoproteins, selenoprotein mRNA expression, fertility, and mortality. J. Biol. Chem. 276: 29798-29804.

Moghadaszadeh B, Beggs AH (2006) Selenoproteins and their impact on human health through diverse physiological pathways. Physiology 21: 307-315.

Morey M, Serras F, Baguna J, Hafen E, Corominas M (2001) Modulation of the Ras/MAPK signalling pathway by the redox function of selenoproteins in Drosophila melanogaster. Dev. Biol. 238: 145-156.

Morozova N, Forry EP, Shahid E, Zavacki AM, Harney JW, Kraytsberg Y, Berry MJ (2003) Antioxidant function of a novel selenoprotein in Drosophila melanogaster. Genes Cells 8: 963-971.

Novoselov SV, Kryukov GV, Xu XM, Carlson BA, Hatfield DL, Gladyshev VN (2007) Selenoprotein H is a nucleolar thioredoxin-like protein with a unique expression pattern. J. Biol. Chem. 282: 11960-11968.

Pan D (2010) The Hippo signaling pathway in development and cancer. Dev. Cell. 19: 491-505.

Rederstorff M, Krol A, Lescure A (2006) Understanding the importance of selenium and selenoproteins in muscle function. Cell Mol. Life Sci. 63: 52-59.

Remmert M, Biegert A, Hauser A, Söding J (2012) HHblits: lightning-fast iterative protein sequence searching by HMM-HMM alignment. Nat. Methods 9: 173-175.

Saraiva MA, Ávila ER, da Silva GF, Macedo GE (2018) Exposure of Drosophila melanogaster to Mancozeb induces oxidative damage and modulates Nrf2 and HSP70/83. Oxid. Med. Cell. Longev. 2018: 5456928.

Shibata T, Ohta T, Tong KI, Kokubu A, Odogawa R, Tsuta K, Asamura H, Yamamoto M, Hirohashi S (2008) Cancer related mutations in NRF2 impair its recognition by Keap1-Cul3 E3 ligase and promote malignancy. Proc. Natl. Acad. Sci. USA. 105: 13568-13573.

Shrotriya S, Deep G, Gu M, Kaur M, Jain AK, Inturi S, Agarwal R, Agarwal C (2012) Generation Of reactive oxygen species by grape seed extract causes irreparable DNA Damage leading to G2/M Arrest and apoptosis selectively in head and neck squamous cell carcinoma cells. Carcinogenesis 33: 848-858.

Singh A, Boldin-Adamsky S, Thimmulappa RK, Rath SK, Ashush H, Coulter J, Blackford A, Goodman SN, Bunz F, Watson WH (2008) RNAi-mediated silencing of nuclear factor erythroid-2-related factor 2 gene expression in nonsmall cell lung cancer inhibits tumor growth and increases efficacy of chemotherapy. Cancer Res. 68: 7975-7984

Speicher T, Foehrenbacher A, Pochic I, Weiland T, Wendel A (2010) Malignant but not naïve hepatocytes of human and rodent origin are killed by TNF after metabolic depletion of ATP by fructose. J. Hepatol. 53: 896-902.

Stagos D, Kazantzoglou G, Magiatis P, Mitaku S, Anagnostopoulos K, Kouretas D (2005) Effects of plant phenolics and grape extracts from Greek varieties of Vitis vinifera on Mitomycin C and topoisomerase I-induced nicking of DNA. Int. J. Mol. Med. 15: 1013-1022.

Sun QA, Kirnarsky L, Sherman S, Gladyshev VN (2001) Selenoprotein oxidoreductase with specificity for thioredoxin and glutathione systems. Proc. Nat. Acad. Sci. USA 98: 3673-3678.
Sun T, Chen QY, Wu LJ, Yao XM, Sun XJ (2012) Antitumor And antimetastatic activities of grape skin polyphenols in a murine model of breast cancer. Food Chem. Toxicol. 50: 3462-3467.

Thevelein, JM, Hohmann S (1995) Trehalose synthase: Guard to the gate of glycolysis in yeast? Trends Biochem. Sci. 20: $3-10$.

Thundimadathil J (2012). Cancer treatment using peptides: current therapies and future prospects. J. Amino Acids 2012: 967347

Tu J, Zhang X, Zhu Y, Dai Y, Li N, Yang F, Zhang Q, Brann DW, Wang X (2015) Cell-permeable peptide targeting the Nrf2-Keap1 interaction: A potential novel therapy for global cerebral ischemia. J. Neurosci. 35: 1472714739.

Tyagi A, Kapoor P, Kumar R, Chaudhary K, Gautam A, Raghava GPS (2013) In Silico models for designing and discovering novel anticancer peptides. Sci. Rep. 3: 2984.

van Can JGP, van Loon LJC, Brouns F, Blaak EE (2012) Reduced glycaemic and insulinaemic responses following trehalose and isomaltulose ingestion: implications for postprandial substrate use in impaired glucose-tolerant subjects. Br. J. Nutr. 108: 1210-1217.

Vicente RL, Spina L, Gómez JPL, Dejean S, Parrou J, François JM (2018) Trehalose-6-phosphate promotes fermentation and glucose repression in Saccharomyces cerevisiae. Microb. Cell. 5: 444-459.

Voelker RA, Langley CH, Leigh-Brown AJ, Ohnishi, S (1978) New data on allozyme loci in Drosophila melanogaster. Drosoph. Inform. Serv. 53: 200.

Wang W, Xiao ZD, Li X, Aziz KE, Gan B, Johnson RL (2015) AMPK modulates Hippo pathway activity to regulate energy homeostasis. Nat. Cell Biol. 17: 490-499.

Warr CG, Shaw KH, Azim A, Piper MDW, Parsons LM (2018) Using mouse and Drosophila models to investigate the mechanistic links between diet, obesity, Type II diabetes, and cancer. Int. J. Mol. Sci. 19: 4110.

Waterhouse A, Bertoni M, Bienert S, Studer G, Tauriello G, Gumienny R, Heer FT, de Beer, TAP, Rempfer C, Bordoli L, Lepore R, Schwede T (2018) SWISS-MODEL: homology modelling of protein structures and complexes. Nucleic Acids Res. 46: W296-W303.

Wei L, Xing P, Su R, Shi G, Ma Z, Zou Q (2017) CPPredRF: a sequence-based predictor for identifying cellpenetrating peptides and their uptake efficiency. J. Proteome Res. 16: 2044-2053.

Xu D, Li X, Shao F, Lv G, Lv H, Lee JH, Qian X, Wang Z, Xia Y, Du L, Zheng Y, Wang H, Lyu J, Lu Z (2019) The protein kinase activity of fructokinase A specifies the antioxidant responses of tumor cells by phosphorylating p62. Sci. Adv. 5: eaav4570.

Yin M, Zhang L (2011) Hippo signaling: A hub of growth control, tumor suppression and pluripotency maintenance. J. Genet. Genom. 38:471-481.

Yoo MH, Xu XM, Carlson BA, Gladyshev VN, Hatfield DL (2006) Thioredoxin reductase 1 deficiency reverses tumor phenotype and tumorigenicity of lung carcinoma cells. J. Biol. Chem. 281: 13005-13008. 
Yoshizane C, Mizote A, Yamada M, Arai N, Arai S, Maruta K (2017) Glycemic, insulinemic and incretin responses after oral trehalose ingestion in healthy subjects. Nutr. J. 16: 9.

Zhang L, Yue T, Jiang J (2009) Hippo signaling pathway and organ size control. Fly 3: 68-73.
Zhou K, Raffoul JJ (2012). Potential anticancer properties of grape antioxidants. J. Oncol. 2012: 803294.

Zhuo P, Diamond AM (2009) Molecular mechanisms by which selenoproteins affect cancer risk and progression. Biochim. Biophys. Acta 1790: 15461554. 